\title{
New Treatments for Achalasia: Novel Ideas, but Are They Ready for Prime Time?
}

\author{
Joel E. Richter
}

Published online: 8 January 2013

(c) Springer Science+Business Media New York 2013

Achalasia is a rare disorder $(1 / 100,000$ people per year) without any age or gender predilection [1]. Patients typically present with dysphagia for solids and liquids, regurgitation of undigested food, nocturnal coughing, chest pain, and weight loss. These symptoms result from impaired, usually absent, peristalsis and incomplete relaxation of the lower esophageal sphincter (LES) leading to stasis of food in the esophagus and esophageal dilation. Why the enteric esophageal neurons gradually disappear in patients with achalasia remains unknown. Evidence is accumulating that an autoimmune response targeted against these neurons, triggered by an infectious agent, possibly HSV-1, may be involved [2]. The diagnosis is made by a combination of tests including barium esophagram, esophageal manometry, and upper endoscopy.

No treatment for achalasia can restore muscular activity to the denervated esophagus; as a consequence, esophageal aperistalsis is rarely reversed. All treatments are thus directed at reducing the gradient across the LES with three goals [3]:

1. Relieving patients symptoms, especially dysphagia and bland regurgitation

2. Improving esophageal emptying

3. Preventing the long term development of megaesophagus.

In the modern era of achalasia treatment, LES disruption is best accomplished by pneumatic dilation using Rigiflex

\footnotetext{
J. E. Richter ( $\square)$

Division of Digestive Diseases and Nutrition, Department of Internal Medicine, Center for Esophageal and Swallowing Disorders, University of South Florida College of Medicine, 12901 Bruce B. Downs Blvd., MDC 72, Tampa, FL 33612, USA e-mail: Jrichte1@health.usf.edu
}

balloons or laparoscopic myotomy, and, less effectively, by pharmacological agents, such as intrasphincteric botulinum toxin injection (Botox) or systemic calcium channel blockers. Symptoms of dysphagia and regurgitation are the easiest to treat; chest pain relief is more unpredictable [4]. Overall, using single or multiple treatment modalities, over $90 \%$ of achalasia patients will do well [5]. Nevertheless, achalasia is never "cured" by our current treatments. Therefore, recurrences will occur requiring "touch up" treatments with higher recurrence rates with longer periods of follow-up. This editorial will review the currently available treatments and potential new endoscopic treatments for achalasia.

Nitrates and calcium channel blockers decrease resting LES pressure in a dose-dependent manner, with a maximum effect of $50 \%$, thereby temporarily relieving dysphagia. These drugs are taken $15-30 \mathrm{~min}$ before meals, but improvement is incomplete and short-lived, efficacy decreases with time and adverse effects (headaches, dizziness, pedal edema) are common [6]. Primary pharmacologic therapy for achalasia now is endoscopic intrasphincteric Botox injection. As a potent inhibitor of acetylcholine release from nerve endings, Botox counteracts the unopposed LES contraction mediated by cholinergic neurons observed in achalasia, helping to reduce LES resting pressure. Botox injections, usually 100-200 U, decrease LES pressure by $50 \%$, markedly improving symptoms in approximately $75 \%$ of achalasia patients. Nonetheless, $50 \%$ or more relapse within 6 months, probably due to regeneration of the presynaptic membranes at the neuromuscular junctions. Best responses, sometimes up to 1.5-2 years after a single Botox injection, are more likely in older patients ( $>60$ years) and those with vigorous achalasia [7]. This treatment may have a cost advantage for elderly patients with a predicted life expectancy of $<2$ years [1]. 
Pneumatic dilation, which disrupts the LES by forceful stretching using air-filled balloons, has become simplified with the advent of the Microinvasive Rigiflex balloon system (Boston Scientific Corp, Natick, MA, USA). These non-compliant polyethylene balloons are available in three diameters $(30,35$, and $40 \mathrm{~mm})$, mounted on a flexible catheter placed over a guidewire at endoscopy. Under fluoroscopic guidance, the balloon is placed across the LES and gradually inflated until the waist, caused by the nonrelaxing LES, is flattened. The pressure required is usually 7-15 psi of air, held for 15-60 s. Most use a graded dilation protocol starting with a $30-\mathrm{mm}$ balloon [8], with subsequent dilations spaced over 4 to 6-week intervals based on symptom relief correlated with repeat LES pressure measurements or assessment of improvement in esophageal emptying [9]. Pneumatic dilation is done in an outpatient setting, with the patient observed for 4-6 h and returning to normal activities the next day. Complications are infrequent with the most serious being esophageal perforation with an overall rate in experienced hands of $2 \%$, of which half will require surgery [10].

In a review of 1,144 patients across 24 studies with an average follow-up of 37 months, Rigiflex pneumatic dilation gave good to excellent symptom relief in a graded fashion in 74,86 , and $90 \%$ of patients treated with 30-, 35-, and 40-mm balloons, respectively [3]. Over a third of patients will have symptom relapse over 4-6 years; however, long-term remission can be achieved in virtually all these patients by repeat dilation according to an "on-demand" strategy based on symptom recurrence [11]. Patients with the best outcomes following pneumatic dilation are older ( $>40$ years), women, and those with a type II pattern by high-resolution manometry [9, 12-14]. Pneumatic dilation is the most cost-effective treatment for achalasia over a 5 to 10 -year post-procedure period [1].

Laparoscopic Heller myotomy is currently the most popular operation for achalasia. Patients are usually hospitalized for less than $48 \mathrm{~h}$, returning to work within 2 weeks. Recent surgical improvements have included extending the myotomy $2-3 \mathrm{~cm}$ onto the proximal stomach in order to cut the gastric sling fibers [15], and the addition of a partial fundoplication to decrease gastroesophageal reflux disease [16]. In a review of 39 studies including nearly 3,100 patients [17], good to excellent symptom relief with laparoscopic myotomy was reported in $89.6 \%$ of subjects over an average follow-up of 35 months. Young patients, especially men and patients with higher LES pressure, may benefit most from primary surgery. Patients failing pneumatic dilation or Botox treatment can be successfully treated with surgical myotomy. Long-term studies suggest deterioration of surgical success over 5-11 years with $18 \%$ requiring pneumatic dilation and 5-10\% repeat myotomy or esophagectomy [1]. Surgical expertise and patient volume are keys to success with most complications (esophageal perforation, incomplete myotomy, death) occurring in the first 50 operations [18]. Surgery is the most costly treatment for achalasia, but may be cost-effective if symptom relief reliably lasts at least 10 years [1].

Only recently has a prospective randomized comparative study been published comparing pneumatic dilation and laparoscopic myotomy performed by physicians skilled in both procedures. The European Achalasia Trial [19] randomized 94 patients to Rigiflex dilation (30 and $35 \mathrm{~mm}$ with up to three repeat dilations allowed) and 106 to laparoscopic myotomy with Dor fundoplication. Both treatments had comparable symptom relief at 2 years: $92 \%$ for dilation and $87 \%$ for myotomy. Barium emptying and LES pressures were improved to similar extent in both groups. This study supports the contention that both treatments are equally effective for up to 2 years, although longer followup will be needed.

Despite the success and safety profile of these two treatments, several new endoscopic treatments for achalasia have been proposed over the last several years: peroral endoscopic myotomy (POEM), removable metal stents, and endoscopic sclerotherapy to the esophagogastric junction (EGJ).

Developed by Inoue in Japan [20], POEM is the most exciting new treatment for achalasia now being widely studied in the US and Europe. An endoscopic myotomy is performed using a submucosal tunnel, the circular muscle fibers are divided over a minimum of $6 \mathrm{~cm}$ in the distal esophagus and $2 \mathrm{~cm}$ onto the cardia as with traditional myotomy, and the mucosal entry site closed with standard endoscopic clips. The procedure is technically demanding, requiring about $2 \mathrm{~h}$. Most patients have postoperative evidence of dissection into the mediastinum or peritoneal cavity with mild transient leukocytosis, although no patients developed fever. Using a validated symptom questionnaire, treatment success was achieved in $94 \%$ of patients with resting] LES pressure falling to an average of $11.8 \mathrm{mmHg}$ within 3 months of follow-up [21]. The excitement of incisionless surgery will need to be balanced against the completeness of the myotomy evaluated by barium emptying or the new Endoflip catheter, which accurately accesses EGJ compliance. Theoretically, a fundoplication is not required, as the surrounding tissues and structures are not dissected during POEM, but recent reports presented at the annual meeting of the American College of Gastroenterology suggest a high rate of postprocedure gastroesophageal reflux [22].

Metal stents are being placed across the EGJ by several centers in China for the treatment of achalasia [23]. The concept is that the stents gradually expand at body temperature over $24 \mathrm{~h}$, resulting in more predictable tearing of the cardia muscle, less scar tissue, and a lower rate of 
restenosis after the stent is removed after 1 month. The simplicity of the procedure is appealing, although enthusiasm is tempered by reported case series not using validated symptom questionnaires, using non-traditional measurements of post-procedure LES pressure, and not assessing esophageal emptying. The overall success seems no better than single-balloon pneumatic dilation, further tempered by a reported rate of stent migration of $20 \%$. Studies performed in esophageal centers outside of China are mandatory to critically evaluate this new procedure.

The most "non-traditional" of these new procedures is endoscopic sclerotherapy with ethanolamine oleate or polidocanol as described by Moreto and colleagues from Spain in this issue of Digestive Diseases and Science [24]. To this reviewer, the concept seems counter-intuitive since sclerosants cause transmural necrosis followed by a variable degree of fibrosis. Endoscopy and injection was performed every 2-4 weeks until dysphagia resolved. All patients had chest pain for several hours following the injections. The reported response was nothing short of phenomenal, with $90 \%$ free of recurrence at 50 months with ethanolamine and $65 \%$ with polidocanol. Nevertheless, this was a telephone follow-up with an unvalidated symptom questionnaire. Although LES pressure decreased impressively from 24.3 to $9.1 \mathrm{mmHg}$, upright esophageal emptying was not reported. Mild strictures responding to $20 \mathrm{~mm}$ dilation were reported in $20 \%$ of the patients, usually those receiving polidocanol. The authors proposed using ethanolamine as the primary sclerosant for treating achalasia owing to better associated clinical outcomes, perhaps due to a more favorable balance between necrosis versus fibrosis. Downsides to this technique include its being labor-intensive, painful, and associated with the development of fibrotic strictures, which might make more traditional treatments like pneumatic dilation or especially myotomy, difficult to perform. Time will tell if other centers can validate these optimistic results and correlate them with state-of-the-art measurements of esophageal function.

Where does this leave the gastroenterologist or general surgeon who infrequently sees more than one case of achalasia a year? Leave it up to the experts and beware of the latter two new endoscopic treatments! The POEM procedure likely will have future clinical application as it applies traditional surgical dogma with an appealing although technically demanding incisionless technique. However, the compromise for a complete myotomy without a fundoplication will be a high rate of reflux diseasethis experiment has been done before and failed [16]. The concept of replacing a non-relaxing LES with a fixed stenosis to open the EGJ either by metal stents or esophageal sclerosants - that is converting achalasia to a peptic stricture-appears to me not as a long-term solution but as a means to generate more endoscopic procedures. Therefore, until more careful studies are performed, achalasia patients will have their best outcomes when evaluated at recognized esophageal Centers of Excellence with staff being knowledgeable about the nuances of esophageal function tests [25] and having experience and high patient volume in performing pneumatic dilation and laparoscopic myotomy with fundoplication.

Conflict of interest None.

\section{References}

1. Richter JE, Boeckxstaens GE. Management of achalasia: surgery or pneumatic dilation. Gut. 2011;60:869-876.

2. Facco M, Brun P, Baesso I, et al. T cells in the myenteric plexus of achalasia patients showed a skewed TCR repertoire and react to HSV-1 antigens. Am J Gastroenterol. 2008;103:1598-1609.

3. Richter JE. Update on the management of achalasia: balloons, surgery and drugs. Expert Rev Gastroenterol Hepatol. 2008;2:435-445.

4. Eckardt VF, Stauf B, Bernhard G. Chest pain in achalasia: patient characteristics and clinical course. Gastroenterology. 1999;116: 1300-1304.

5. Vela MF, Richter JE, Washberger D, et al. Complexities of managing achalasia at a tertiary referral center: use of pneumatic dilation, Heller myotomy and botulinum toxin injection. Am J Gastroenterol. 2004;99:1029-1036.

6. Hoogerwerf W, Pasricha PJ. Pharmacologic therapy in treating achalasia. Gastrointest Endosc Clin North Am. 2001;11:311-323.

7. Pasricha PJ, Rai R, Ravich WJ, et al. Botulinum toxin for achalasia: long-term outcome and redictors of response. Gastroenterology. 1996;110:1410-1415.

8. Kadakia SC, Wong RK. Graded pneumatic dilation using Rigiflex achalasia dilators in patients with primary esophageal achalasia. Am J Gastroenterol. 1993;103:1732-1738.

9. Vela MF, Ricther JE, Khandwala E, et al. The long-term efficacy of pneumatic dilation and Heller myotomy for the treatment of achalasia. Clin Gastroenterol Hepatol. 2006;4:580-587.

10. Katzka DA, Castell DO. Review article: an analysis of the efficacy, perforation rates and methods used in pneumatic dilation for achalasia. Alim Pharmacol Therap. 2011;34:832-839.

11. Zerbid F, Thetiot V, Ricky F, et al. Repeated pneumatic dilations as long-term maintenance therapy for esophageal achalasia. Am J Gastroenterol. 2006;101:692-697.

12. Vantrappen G, Helemans J, Deloof W, et al. Treatment of achalasia with pneumatic dilation. Gut. 1971;12:268-275.

13. Vaezi MF, Baker MF, Achkar E, Richter JE. Timed barium esophagram: better predictor of long-term success after pneumatic dilation than symptom assessment. Gut. 2002;50:765-770.

14. Pandolfino JE, Kwiatek MA, Nealis T, et al. Achalasia: it's not all one disease-subclassification by high-resolution manometry. Gastroenterology. 2008;135:1383-1391.

15. Oelschager BK, Chang L, Pellegrini CA. Improved outcomes after extended gastric myotomy in achalasia. Arch Surg. 2003; 139:490-495.

16. Richards W, Torquati A, Holzman M, et al. Heller myotomy vs Heller myotomy with Dor fundoplication for achalasia: a prospective randomized double-blind clinical trial. Ann Surg. 2004; 240:405-415.

17. Campos GM, Vittinghoff E, Rabi C, et al. Endoscopic and surgical treatment for achalasia. A systemic review and metal-analysis. Ann Surg. 2009;249:45-57. 
18. Sharp KW, Khaitan L, Scholz S, et al. 100 consecutive minimally invasive Heller myotomies: lessons learned. Ann Surg. 2002;235: 631-639.

19. Boeckxstaens GE, Annese V, Bruley des Varannes S, et al. Pneumatic dilation vs laparoscopic Heller myotomy for idiopathic achalasia. N Eng J Med. 2011;364:1807-1816.

20. Inoue $\mathrm{H}$, Minami $\mathrm{H}$, Kobayashi $\mathrm{Y}$, et al. Peroral endoscopic myotomy (POEM) for esophageal achalasia. Endoscopy. 2010;42: 265-271.

21. Renteln D, Inoue H, Minami H, et al. Peroral endoscopic myotomy for the treatment of achalasia: a prospective single center study. Am J Gastroenterol. 2012;107:411-417.

22. Stavropoulos S, Brathwaite C, Halwon B, et al. POEM: effective NOTES accessible to the gastroenterologist, 3-year experience at US Center. Am J Gastroenterol. 2012;107(Suppl):S1909.
23. Cheny YS, Ma F, Li YD, et al. Temporary self-expanding metallic stents for achalasia: a prospective study with long-term follow-up. World J Gastroenterol. 2010;16:5111-5117.

24. Moreto M, Ojembarrena E, Bareuren A, Casedo I. Treatment of achalasia by injection of scerlosant substances: a long term report. Dig Dis Sci. 2012. (Epub ahead of print). doi:10.1007/ s10620-012-2476-x.

25. Richter JE. The diagnosis and misdiagnosis of achalasia: it does not have to be so difficult. Clin Gastroenterol Hep. 2011;9: 1010-1011. 